Journal of Bangladesh Academy of Sciences, Vol. 35, No. 2, 211-220, 2011

\title{
DIELECTRIC BEHAVIOR OF BINARY SOLUTIONS OF THERMACOL IN ORGANIC SOLVENTS
}

\author{
N.P. ADHIKARI* , P. BHATTARAI ${ }^{1}$, M.M. ARYAL AND D.R. MISHRA \\ Central Department of Physics, Tribhuvan University, Kirtipur, Kathmandu, Nepal
}

\begin{abstract}
The static dielectric constant and dielectric loss for the binary solutions of thermacol in various organic solvents have been measured varying the concentration of solute. The dielectric constant varied linearly with the concentration of solute, whereas the dielectric loss showed excess peak at a certain concentration of the solute for binary solutions. The authors also performed the first-principles calculations to study the equilibrium configurations of ethyl acetate and n-butyl acetate and estimated dipole moments. The experimental values of dipole moment for ethyl acetate and n-butyl acetate agreed fairly well with those calculated from first-principles.
\end{abstract}

Key words: Dielectric constant, Dielectric loss, Binary solution, Dipole moment

\section{INTRODUCTION}

Dielectric properties of any material is important from practical as well as fundamental point of view. The majority of polymers are typical dielectric materials. The dielectric behavior of polymers is mainly determined by their physical and chemical structure and composition. Some of the important dielectric properties are the dielectric constant, dielectric loss and the electric break down strength. The term dielectric behavior usually refers to the variation in these properties of a material as a function of frequency, composition, voltage, pressure and temperature. In general, dielectric constant of an n-component solution is expected to lie within the values as given by Wiener inequalities (Tareev 1975). Any deviation of the observed dielectric constant of an n-component solution from these limits could be attributed to the solute-solvent interactions occurring in the solution.

In the present work, the authors studied the concentration dependence of the static dielectric constant and the dielectric loss for the binary solutions of thermacol, a nonpolar polymer of low density, in ethyl acetate and n-butyl acetate with varying concentration of thermacol ranging up to about $85 \%$ by volume (Riajl 2004, Niraula 2004). Ethyl acetate was obtained from Merck Fine Chemicals, whereas, and n-butyl acetate from Laboratory Rassayan Ltd., India. The measurements have also been carried to study dielectric constant and dielectric loss for the binary solution of toluene in ethyl acetate. Toluene was obtained from Qualigens Fine Chemicals. The researchers have used Schering bridge method for the measurement of static dielectric constant and

*Corresponding author: <npadhikari@gmail.com>. 
dielectric loss (Hague 1955). With these data, the dielectric constant for thermacol and dipole moments for ethyl acetate and n-butyl acetate was estimated.

Further, the authors performed first principles calculations to calculate equilibrium configuration of ethyl acetate, n-butyl acetate, ethyl acetate and styrene, n-butyl acetate and styrene, and, toluene and styrene. The dipole moments for ethyl acetate and n-butyl acetate were also calculated. The data are compared with those from the experiments. The binding energies for ethyl acetate and styrene, n-butyl acetate and styrene, and toluene and styrene have also been calculated.

\section{FIRST PRINCIPLES CALCULATIONS: THEORY}

First-principles ( $a b$ initio) methods are being widely used to study the electronic structure of solids, surfaces, clusters, complex systems etc. Ohno et al. (1999). Recently, the applications of first principles calculations have gained much prominence in the ever expanding field of material science including polymers. To test experimental results in which analytical calculations is not possible, it is one of the most reliable tools.

There are many ab initio methods. Hartree-Fock self-consistent method is based on the one-electron approximation in which the motion of each electron in the effective field of all the other electrons is governed by a one-particle Schrodinger equation. As the antisymmetric product of one-electron wave functions is used in the Hartree-Fock approximation, it takes into account of the correlation arising due to electrons of the same spin, however, the motion of the electrons of the opposite spin remains uncorrelated in this approach.

In density functional theory (DFT) method, the exchange-correlation is expressed as a functional of the electron density and the electronic states are solved for selfconsistency as in the Hartree-Fock approximation. The exchange-correlation potential not only includes the exchange interaction but also the dynamic correlation effect arising due to the Coulomb repulsion between the electrons. In principle, DFT is exact, however, in practice, both exchange and dynamic correlation effects are treated approximately. In DFT, following effective one-electron Schrodinger equation is solved for an $\mathrm{i}^{\text {th }}$ electron,

$-\frac{h^{2}}{16 \pi^{2} m} \nabla_{i}^{2} \psi_{\lambda}(\mathbf{r})-\sum_{j} \frac{e^{2} Z_{j}}{\left|\mathbf{r}_{i}-\mathbf{R}_{j}\right|} \psi_{\lambda}(\mathbf{r})+\int \frac{\rho\left(\mathbf{r}_{j}\right)}{\left|\mathbf{r}_{i}-\mathbf{r}_{j}\right|} \psi_{\lambda}(\mathbf{r}) d \mathbf{r}_{j}+V_{x c}[\rho](\mathbf{r}) \psi_{\lambda}(\mathbf{r})=\varepsilon_{\lambda} \psi_{\lambda}(\mathbf{r})$

where, $h$ is Planck's constant, $m$ is the mass of an electron, $e$ is the charge of an electron, $Z_{j}$ is the atomic number of nucleus at position $\boldsymbol{R}_{\boldsymbol{j}}$ and $\boldsymbol{r}_{i}$ is the position of $\mathrm{i}^{\text {th }}$ electron. In Eq. (1), the first term in left hand side is the kinetic energy of $i^{\text {th }}$ electron, and the second term represents the interaction energy of $i^{\text {th }}$ electron to nuclei. The third term is the electrostatic energy of the electron in the field generated by the total electron density $\rho(\mathbf{r})$. The fourth term contains the many-body effects, lumped together in an exchange- 
correlation potential. In the present work, the researchers considered DFT method and used generalized gradient approximation to calculate the exchange correlation term Parr and Yang (1989). The DFT calculations have been carried out using the Gaussian 98 set of programs.

\section{RESULTS AND DISCUSSION}

Fig. 1 shows the dielectric constant $(\varepsilon)$ as a function of the volume fraction $(\varphi)$ of thermacol for the binary solutions of (i) thermacol in ethyl acetate and (ii) thermacol in n-butyl acetate at a temperature $298 \mathrm{~K}$. It is seen from Fig. 1 that with the increase of the concentration of thermacol, the dielectric constant of the solution decreases linearly, which is expected because increasing the concentration of thermacol is to increase the concentration of non-polar solute in the binary solution. The best fitted lines are,

$$
\begin{aligned}
& \varepsilon=5.93-3.9 \varphi \\
& \varepsilon=4.95-2.98 \varphi
\end{aligned}
$$

for thermacol/ethyl acetate and thermacol/butyl acetate solutions, respectively. Assuming that Eqs. (2) and (3) to be valid over the whole range of concentration of thermacol, authors have estimated the dielectric constant of thermacol by extrapolating Eqs. (2) and (3) for $\varphi=1$. It is seen that the values of dielectric constant for thermacol from Eqs. (4) and (5) are close to each other with $\varepsilon=1.98 \pm 0.04$. Fig. 2 shows the dielectric constant

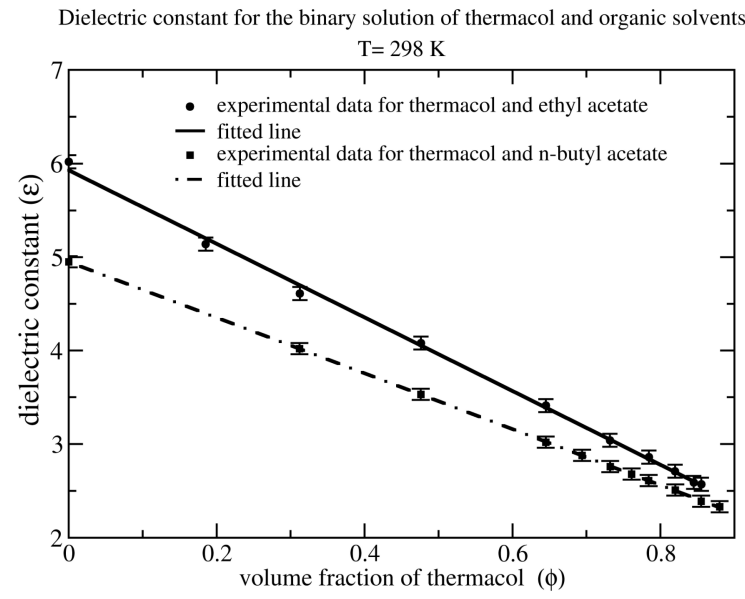

Fig. 1. Dielectric constant of thermacol/ethyl acetate and thermacol/n-butyl acetate binary solutions as a function of volume fraction of thermacol at temperature $298 \mathrm{~K}$.

of toluene and ethyl acetate binary solution as a function of concentration of toluene. It is clearly seen from Figs 1 and 2 that the dielectric constant of thermacol/ethyl acetate and toluene/ethyl acetate follows linear behavior as a function of volume fraction of nonpolar solute, i.e. thermacol and toluene, respectively. 
Dielectric constant for toluene and ethyl acetate binary solution

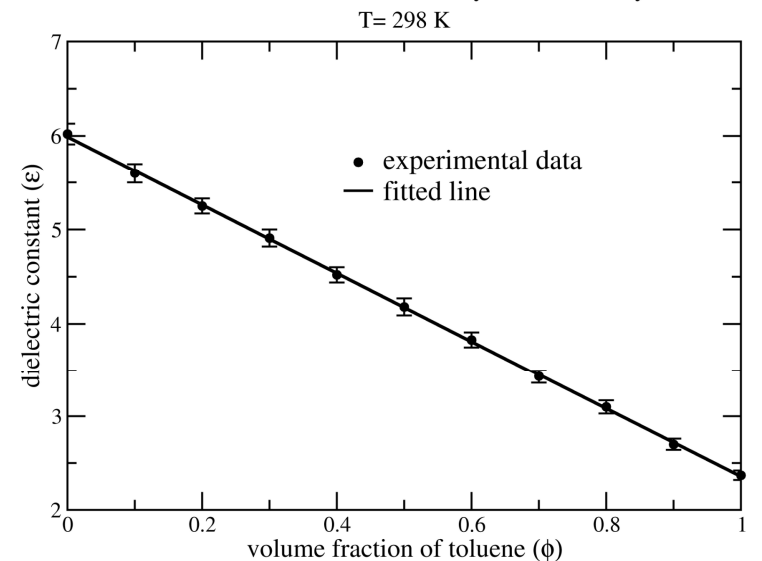

Fig. 2. Dielectric constant of toluene/ethyl acetate binary solution as a function of volume fraction of toluene at temperature $298 \mathrm{~K}$.

Fig. 3 shows the refractive index $(\mu)$ as a function of the volume fraction of thermacol for the binary solutions of (i) thermacol in ethyl acetate and (ii) thermacol in n-butyl acetate at a temperature $298 \mathrm{~K}$. It is seen from Fig. 3 that the refractive index of the solution increases linearly as the volume fraction of thermacol increases and can be expressed as,

$$
\begin{aligned}
& \mu=1.371+0.032 \phi \\
& \mu=1.390+0.010 \varphi
\end{aligned}
$$

Refractive index for the binary solution of thermacol in organic solvents

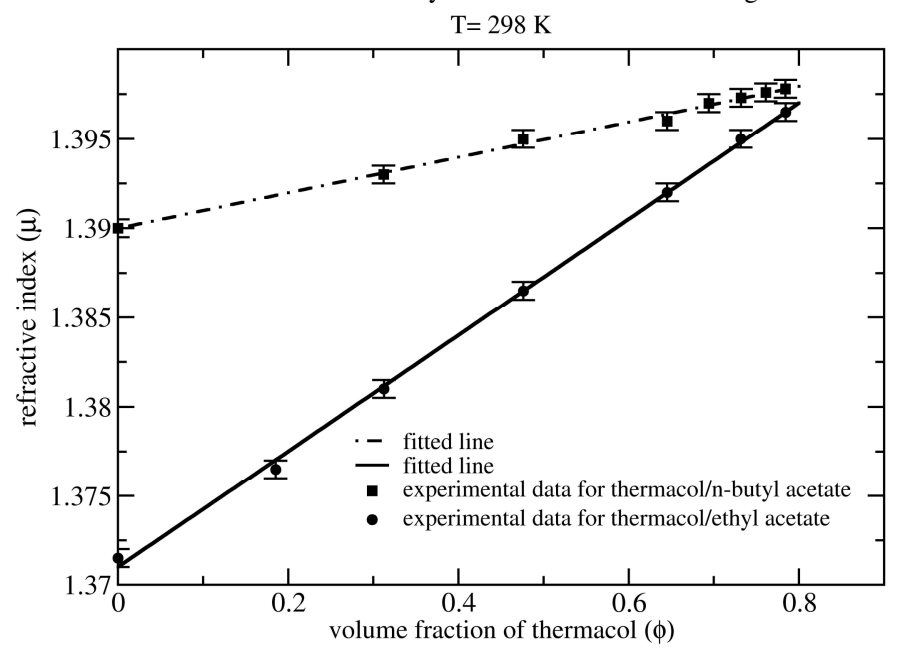

Fig. 3. Refractive index of thermacol/ethyl acetate and thermacol/n-butyl acetate binary solutions as a function of volume fraction of thermacol at temperature $298 \mathrm{~K}$. 
for thermacol/ethyl acetate and thermacol/butyl acetate binary solutions respectively. Assuming that Eqs. 4 and 5 to be valid over the whole range of concentration of thermacol, authors have estimated the refractive index of thermacol for $\varphi=1$. It is seen that the values of refractive index for thermacol from Eqs. (4) and (5) are close to each other with $\mu=1.40 \pm 0.01$, and the relation $\varepsilon \approx \mu^{2}$ holds for thermacol indicating that thermacol is a non-polar polymer.

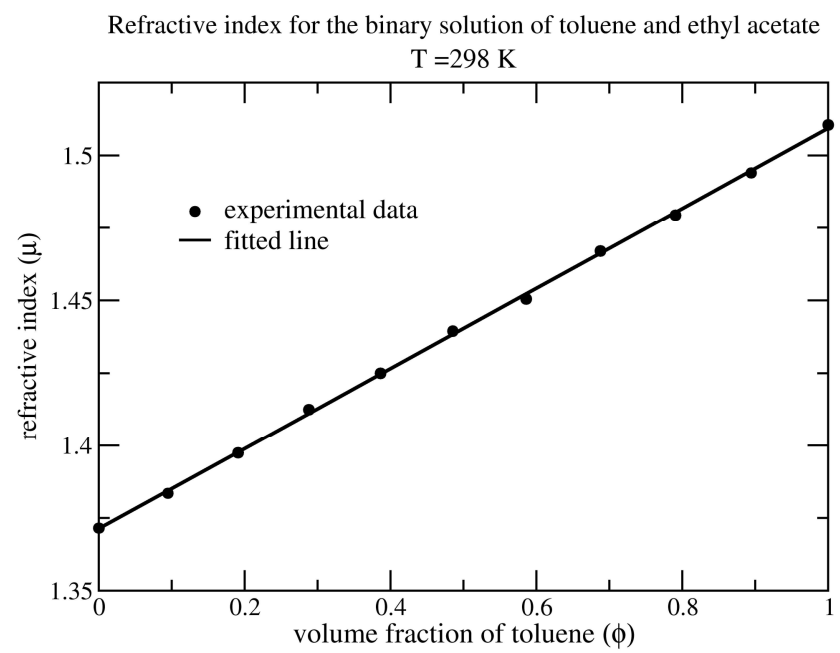

Fig. 4. Refractive index of toluene/ethyl acetate binary solution as a function of volume fraction of toluene at temperature $298 \mathrm{~K}$.

Fig. 4 shows the refractive index for toluene/ethyl acetate binary solution at a temperature $298 \mathrm{~K}$ as a function of volume fraction of toluene. It is clearly seen from Figs. 3 and 4 that the refractive index of thermacol/ethyl acetate and toluene/ethyl acetate follows linear behavior as a function of volume fraction of thermacol and toluene, respectively.

Fig. 5 shows the dielectric loss at frequency $12 \mathrm{kHz}$ for toluene/ethyl acetate binary solution at $298 \mathrm{~K}$ and Fig. 6 shows the dielectric loss at frequency $12 \mathrm{kHz}$ for thermacol/ethyl acetate and thermacol/butyl acetate binary solutions at $298 \mathrm{~K}$. It is seen from Fig. 5 that the dielectric loss decreases with increasing concentration of non-polar solute.

However, it is seen from Fig. 6 that the dielectric loss for both polymer solutions increases with increasing concentration of thermacol up to about $6 \mathrm{gm}$ of thermacol in $100 \mathrm{cc}$ of the solvent used and then decreases on further increasing the concentration of thermacol. In heterogeneous dielectrics the observed dielectric loss is given by Smyth (1955),

$$
\tan \delta_{\mathrm{obs}}=\tan \delta_{\mathrm{dc}}+\tan \delta_{\mathrm{mw}}+\tan \delta_{\text {debye }}
$$




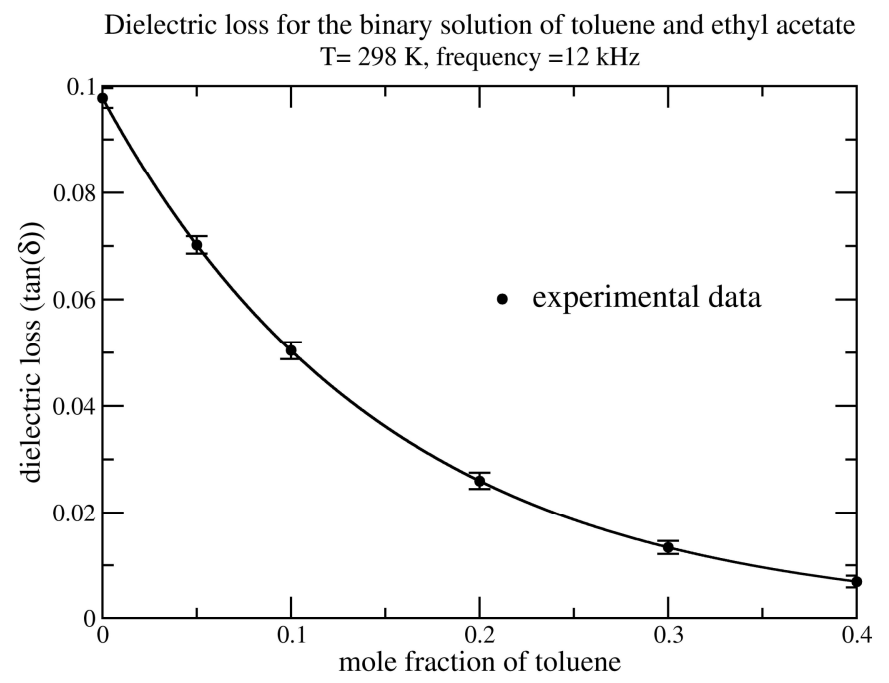

Fig. 5. Dielectric loss of toluene/ethyl acetate binary solution as a function of volume fraction of toluene at temperature $298 \mathrm{~K}$.

where, $\tan \delta_{\mathrm{dc}}$ is the loss factor due to conductivity, $\tan \delta_{\mathrm{mw}}$ is the Maxwell-Wagner loss factor or loss due to interfacial polarization and $\tan \delta_{\text {debye }}$ is the loss due to dipolar polarization. The estimation of the dipolar contribution to the dielectric loss, in the frequency range studied, shows that it is of the order of $10^{-6}$ which is less than $1 \%$ of the observed dielectric loss.

Dielectric loss for the binary solution of thermacol in organic solvents

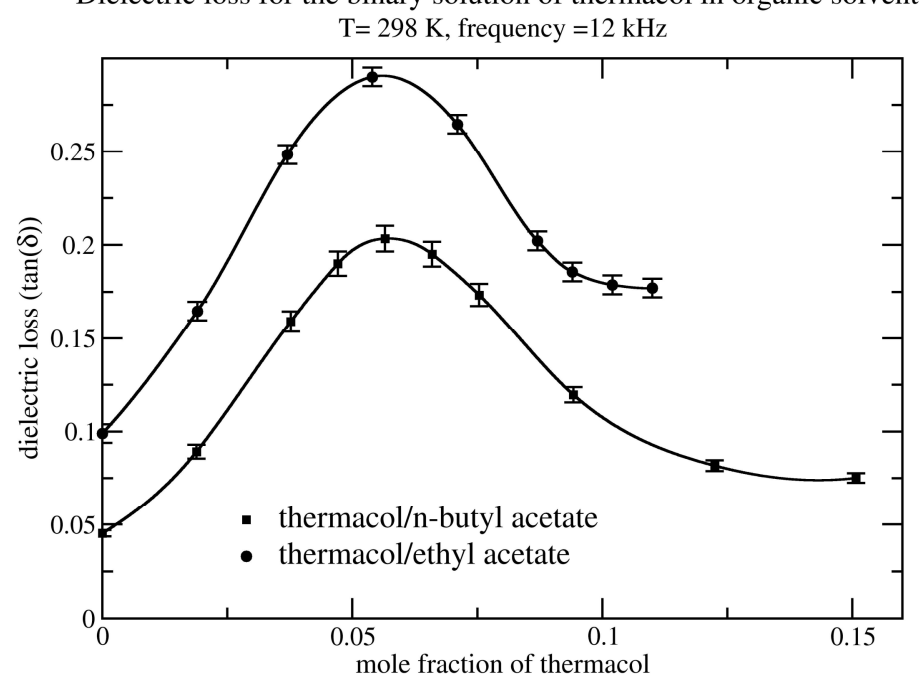

Fig. 6. Dielectric loss of thermacol/ethyl acetate and thermacol/n-butyl acetate binary solutions as a function of volume fraction of thermacol at temperature $298 \mathrm{~K}$. 
The dielectric loss due to conductivity is generally expected to decrease with the addition of a non-polar component to polar dielectrics as observed in Fig. 5 for toluene/ethyl acetate solution. The observed dielectric loss as a function of concentration of thermacol cannot be explained only considering $\tan \delta_{\mathrm{dc}}$ and $\tan \delta_{\text {debye }}$. Hence, we need to consider the dielectric loss due to interfacial polarization which arises when two phases of solution differ from each other in dielectric constant and conductivity in order to explain the observed dielectric loss in binary solution of thermacol in ethyl acetate or in n-butyl acetate.

The authors also estimated the values of dipole moment of ethyl acetate and n-butyl acetate using Onsager relation. From Onsager relations (7) authors have,

$$
\frac{\left(2 \varepsilon+\mu^{2}\right)\left(\varepsilon-\mu^{2}\right) M}{\varepsilon\left(2+\mu^{2}\right)^{2} \rho}=\frac{4 \pi N_{A} p^{2}}{9 k_{B} T}
$$

where $M$ is the molecular weight, $\rho$ is the density, $N_{A}$ is Avogadro's number and $k_{B}$ is the Boltzmann constant and $p$ is the dipole moment. Figs. 7 and 8 show $\left[\left(2 \varepsilon^{+} \mu^{2}\right)\right.$ $\left.\left(\varepsilon-\mu^{2}\right) \mathrm{M}\right] /\left[\varepsilon\left(2+\mu^{2}\right) \rho\right]$ as a function of inverse of temperature for ethyl acetate and n-butyl acetate respectively. From Figs. 7 and 8 , it can be seen that $\left[\left(2 \varepsilon+\mu^{2}\right)\left(\varepsilon-\mu^{2}\right) \mathrm{M}\right] /\left[\varepsilon\left(2+\mu^{2}\right) \rho\right]$ varies linearly as a function of inverse of temperature as expected.

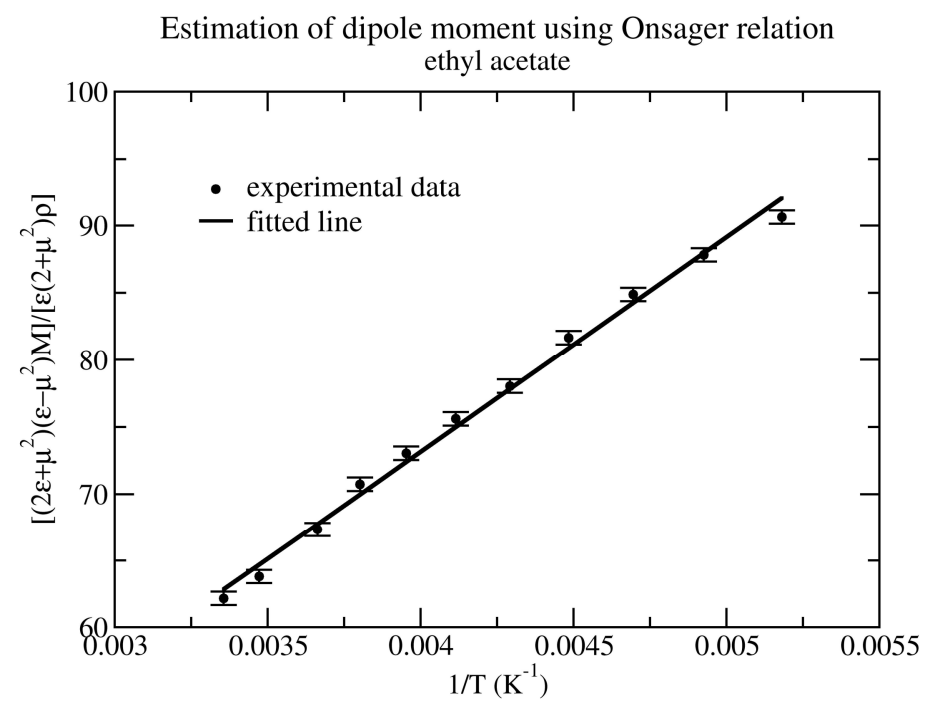

Fig. 7. $\left[\left(2 \varepsilon+\mu^{2}\right)\left(\varepsilon-\mu^{2}\right) \mathrm{M}\right] /\left[\varepsilon\left(2+\mu^{2}\right) \rho\right]$ for thermacol and ethyl acetate binary solution as a function of inverse of temperature.

The dipole moment of ethyl acetate and n-butyl acetate estimated from the slope of the fitted lines in Figs. 7 and 8 turn out to be 1.72 and 1.81 debye, respectively. The dipole moments have also been estimated from Debye's method of mixtures. The dipole moments for ethyl acetate and n-butyl acetate, estimated from Debye's method of 